\title{
Infrared massive gluon propagator from a BRST-invariant Gribov horizon in a family of covariant gauges
}

\author{
Bruno W. Mintz, Leticia F. Palhares, Giovani Peruzzo, and Silvio P. Sorella \\ UERJ-Universidade do Estado do Rio de Janeiro, Departamento de Física Teórica, \\ Rua São Francisco Xavier 524, 20550-013 Maracanã, Rio de Janeiro, Brasil
}

(Received 20 December 2018; published 11 February 2019)

\begin{abstract}
A distinctive feature observed in lattice simulations of confining non-Abelian gauge theories, such as quantum chromodynamics, is the presence of a dynamical mass for the gauge field in the low-energy regime of the theory. In the Gribov-Zwanziger framework in the Landau gauge, such mass is a consequence of the generation of the dimension-two condensates $\left\langle A_{\mu}^{a} A_{\mu}^{a}\right\rangle$ and $\left\langle\bar{\varphi}_{\mu}^{a b} \varphi_{\mu}^{a b}-\bar{\omega}_{\mu}^{a b} \omega_{\mu}^{a b}\right\rangle$, where $A$ is the gluon field and the fields $\bar{\varphi}, \varphi, \bar{\omega}$, and $\omega$ are Zwanziger's auxiliary fields. In this work, we show that, in the recently developed BRST-invariant version of the refined Gribov-Zwanziger theory, these condensates can be introduced in a BRST-invariant way for a family of $R_{\xi}$ gauges. Their values are explicitly computed to first order and turn out to be independent of the gauge parameters contained in the gauge-fixing condition, as expected from the BRST invariance of the formulation. This fact supports the possibility of a gauge-parameter independent nonzero infrared gluon mass, whose value is the same as the one in the Landau gauge.
\end{abstract}

DOI: 10.1103/PhysRevD.99.034002

\section{INTRODUCTION}

For more than fifty years, the standard way to perform the quantization of a gauge field theory in the continuum has been the Faddeev-Popov procedure [1]. One of the main features of this approach is that the quantization is effectively performed only if some given gauge condition is imposed. The most popular choices of gauge conditions in the study of the strong interaction include not only covariant and colorsymmetric gauges, such as the Landau, the linear covariant, and the $R_{\xi}$ gauges [2,3], but also noncovariant ones, such as the Coulomb gauge [4-6]. Another category of gauges includes those in which color symmetry is not explicitly enforced, such as the maximal Abelian gauge [7-9]. For an interesting discussion about several issues that may appear in the use of different gauges, see [10].

Each of these gauge choices can, at least in principle, facilitate the understanding of some given physical aspect of the theory. In any case, one expects that the actual physical observables do not depend on the choice of the gauge condition, so that every gauge choice should lead to the same physical results. Although this has been shown to be true in perturbative calculations, the proof of gauge

Published by the American Physical Society under the terms of the Creative Commons Attribution 4.0 International license. Further distribution of this work must maintain attribution to the author(s) and the published article's title, journal citation, and DOI. Funded by SCOAP . equivalence is not as straightforward at the nonperturbative level. Finally, although it is possible to successfully formulate a gauge-invariant version of Yang-Mills theory on a discretized spacetime in the nonperturbative regime [11], continuum approaches still rely on the aforementioned gauge-fixing procedure.

As discussed by Gribov in his seminal work [12], the Faddeev-Popov procedure is consistent only at the perturbative level. For definiteness, let us consider the Landau gauge [13]. As one considers physical processes at lower and lower energies, the strong coupling becomes larger and the gauge field configurations may depart from the vicinity of $A=0$. As a result, the Faddeev-Popov operator $[\mathcal{M}(A)]^{a b}$ in the Landau gauge, $\partial_{\mu} A_{\mu}^{a}=0$,

$$
[\mathcal{M}(A)]^{a b}=-\partial_{\mu} D_{\mu}^{a b}=-\delta^{a b} \partial^{2}+g f^{a b c} A_{\mu}^{c} \partial_{\mu},
$$

whose determinant appears in the path integral formulation, acquires zero modes, i.e., eigenfunctions with vanishing eigenvalues, rendering thus the quantization procedure ill defined. The presence of these zero modes indicates that some gauge copies were not removed by the FaddeevPopov procedure. In other words, the gauge-fixing procedure has not fully eliminated the multiple counting of physical states in the path integral due to gauge invariance. This is called the Gribov problem.

A solution to the Gribov problem would be to evaluate the path integral in such a way that only one representative for each gauge orbit is accounted for. This subset of the 
gauge field configuration space is the fundamental modular region, whose practical implementation has not yet been achieved. In order to take into account the existence of the gauge copies, a partial solution was proposed by Gribov himself in the Landau gauge, amounting to constrain the functional integration over the gauge fields to a subset of all field configurations so that the Faddeev-Popov operator $\mathcal{M}$, Eq. (1), has only positive eigenvalues. This subset is called the Gribov region, and its boundary is called the Gribov horizon.

A practical way to implement the restriction of the path integral to the Gribov region has been proposed by Zwanziger in [14]. The overall procedure can be seen as the use of the Lagrange multiplier method with the addition of an extra term to the action to enforce the constraint of the Gribov region. This term is the horizon function, given by

$$
\begin{aligned}
\gamma^{4} H(A)= & \gamma^{4} g^{2} \int d^{d} x d^{d} y f^{a b c} A_{\mu}^{b}(x)\left[\mathcal{M}(A)^{-1}\right]^{c e}(x, y) \\
& \times f^{a d e} A_{\mu}^{d}(y)
\end{aligned}
$$

in the Landau gauge, where $\gamma$ is the Gribov parameter, which is not a free parameter, but rather fixed selfconsistently from a gap equation, called the horizon condition, see $[14,15]$. In an initially scale-invariant theory, as is the case of Landau-gauge Yang-Mills, the Gribov parameter can be shown to be proportional to the renormalization-group invariant scale that appears as a consequence of conformal anomaly, leading to a nonperturbative infrared effective action, known as the Gribov-Zwanziger action. Since (2) contains the inverse of a field-dependent differential operator, the horizon function is a nonlocal functional of the fields. In order to write the resulting effective field theory with the horizon constraint as a local field theory, it is necessary to introduce auxiliary bosonic fields, $\bar{\varphi}$ and $\varphi$, and fermionic fields, $\bar{\omega}$ and $\omega$ [14,15]. With these ingredients, one can write down the GribovZwanziger (GZ) action in local form in Euclidean space for the Landau gauge, namely

$$
S_{\mathrm{GZ}}=S_{\mathrm{FP}}+S_{h},
$$

where

$$
S_{\mathrm{FP}}=\int d^{d} x\left[\frac{1}{4} F_{\mu \nu}^{a} F_{\mu \nu}^{a}+i b^{a} \partial_{\mu} A_{\mu}^{a}+\bar{c}^{a} \partial_{\mu} D_{\mu}^{a b} c^{b}\right]
$$

is the Faddeev-Popov Lagrangian in the Landau gauge and

$$
\begin{aligned}
S_{h}= & \int d^{d} x\left[\bar{\varphi}_{\mu}^{a b}[\mathcal{M}(A)]^{a c} \varphi_{\mu}^{c b}-\bar{\omega}_{\mu}^{a b}[\mathcal{M}(A)]^{a c} \omega_{\mu}^{c b}\right. \\
& \left.+i g \gamma^{2} f^{a b c} A_{\mu}^{a}\left(\varphi_{\mu}^{b c}+\bar{\varphi}_{\mu}^{b c}\right)\right]
\end{aligned}
$$

is the local version of the horizon function in terms of Zwanziger's auxiliary fields.
An interesting feature of the gluon propagator in the GZ framework is that it violates reflection positivity, which is one of the Osterwalder-Schrader axioms of Euclidean field theory $[16,17]$. Actually, the so-called positivity violation has been interpreted as a sign of confinement [18-20]. However, the gluon GZ propagator still lacks an important feature: according to several results from different nonperturbative methods-such as lattice numerical simulations, functional renormalization group and DysonSchwinger equations - the gluon propagator should be finite at zero momentum and the ghost propagator should behave as $p^{-2}$ as one approaches the infrared. This is known as the decoupling solution [21-29]. The GZ gluon propagator, on its turn, vanishes in the infrared and the ghost propagator behaves as $p^{-4}$ for $p \rightarrow 0$, which is called the scaling behavior [20]. This is an indication that the GZ action is still plagued by some nonperturbative instability. In fact, it turns out that the condensation of mass dimension $d_{m}=2$ operators, such as $A^{2}$ and $\langle\bar{\varphi} \varphi-\bar{\omega} \omega\rangle$ takes place $[26,30]$, resulting in a gluon propagator which is nonvanishing at $p=0$. Since these condensates are nonzero, it is possible to include them directly in the infrared action, which is equivalent to give mass to the gluon and to the Zwanziger auxiliary fields. The resulting action is called the refined Gribov-Zwanziger (RGZ) theory in the Landau gauge:

$$
\begin{aligned}
S_{\mathrm{RGZ}}= & S_{\mathrm{FP}}+S_{h} \\
& +\int d^{d} x\left[\frac{m^{2}}{2} A_{\mu}^{a} A_{\mu}^{a}+M^{2}\left(\bar{\varphi}_{\mu}^{a b} \varphi_{\mu}^{a b}-\bar{\omega}_{\mu}^{a b} \omega_{\mu}^{a b}\right)\right] .
\end{aligned}
$$

In spite of the success of the RGZ approach in the Landau gauge, its original formulation does not allow for an extension to other gauges such as the linear covariant gauges or the $R_{\xi}$ gauges. The main reason for that is the lack of BRST invariance of both GZ and RGZ actions, Eqs. (3) and (6), respectively. Recently, however, an equivalent formulation of the theory was developed which possesses full BRST invariance, making the extension to other gauges possible [31]. Indeed, some features of the GZ and the RGZ theories in the linear covariant gauge have already been explored, for example, in [32-34]. Furthermore, as discussed in [35], the horizon function of a large class of covariant and color invariant gauge conditions, which are continuously connected to the Landau gauge, can be expressed in a unified manner. As a consequence of this new formulation, correlation functions of gauge invariant operators become independent of gauge parameters and are given by their values in the Landau gauge [35].

In this work, we explore the instability of the GribovZwanziger theory that gives rise to the refined GZ theory in a gauge-parameter independent manner. We do this by 
considering a $R_{\xi}$ gauge, which can be understood as a generalization of the linear covariant gauge. In Sec. II, we review the BRST invariant local formulation of the GribovZwanziger effective theory [32] and formulate it in the $R_{\xi}$ gauge. Next, in Sec. III, we calculate the vacuum energy in the GZ effective theory at leading order and show that it is independent of the gauge parameters. Finally, in Sec. IV, we show that the gauge invariant dimension-two condensates $\left\langle A^{h} A^{h}\right\rangle$ and $\langle\bar{\varphi} \varphi-\bar{\omega} \omega\rangle$ are generated by the GZ dynamics, leading to the finite mass terms present in the RGZ effective theory. Final remarks are gathered in Sec. V.

\section{THE BRST-INVARIANT GRIBOV-ZWANZIGER FRAMEWORK}

According to the gauge principle, a crucial step towards a well-defined theory at the quantum level is the establishment of the BRST invariance of the action. Let us briefly review the BRST-invariant formulation of the GribovZwanziger action, which will allow us to extend previous results in the Landau gauge to a family of other gauges continually connected to it. Following [32,36,37], we start by introducing a composite $S U(N)$ gauge field

$$
\left(A^{h}\right)_{\mu}=h^{\dagger} A_{\mu} h+\frac{i}{g} h^{\dagger} \partial_{\mu} h,
$$

where

$$
h(x)=\exp \left(i g \xi^{a}(x) T^{a}\right)
$$

is a matrix valued field, with $T^{a}\left(a=1, \ldots, N^{2}-1\right)$ being the generators of $S U(N)$ and the field $\xi$ is related to the well-known Stueckelberg field [37]. With such a definition, it is possible to define a consistent nilpotent set of BRST transformations such that $s\left(A^{h}\right)_{\mu}^{a}=0$, as described in more details in the Appendix.

An important property of the field $A^{h}$ is that, in the Landau gauge, it may be effectively replaced by the gauge field $A$ in any perturbative calculation, since the Stueckelberg field propagator $\left\langle\xi^{a}(p) \xi^{b}(-p)\right\rangle$ vanishes in this case [32]. In addition, the BRST invariant field $A^{h}$ field can be employed to obtain a BRST invariant expression for Zwanziger's horizon function [31,32], namely

$$
\begin{aligned}
S_{h}^{\prime}= & \int d^{d} x\left[\bar{\varphi}_{\mu}^{a b}\left[\mathcal{M}\left(A^{h}\right)\right]^{a c} \varphi_{\mu}^{c b}-\bar{\omega}_{\mu}^{a b}\left[\mathcal{M}\left(A^{h}\right)\right]^{a c} \omega_{\mu}^{c b}\right. \\
& \left.+i g \gamma^{2} f^{a b c}\left(A^{h}\right)_{\mu}^{a}\left(\varphi_{\mu}^{b c}+\bar{\varphi}_{\mu}^{b c}\right)\right],
\end{aligned}
$$

whose sole difference with respect to (2) is the replacement $A \rightarrow A^{h}$. Due to the properties of the field $A^{h}$, both expressions (5) and (9) turn out to be equivalent in the Landau gauge. Given the BRST invariance of $A^{h}$, it is consistent to define the auxiliary fields $\bar{\varphi}, \varphi, \bar{\omega}$, and $\omega$ as
BRST singlets, so that the new horizon function (9) is itself BRST invariant.

This now allows us to generalize the GZ theory in other gauges different from the Landau gauge, i.e., the linear covariant gauge $[31,33,38,39]$, and the more general family of covariant, color-preserving gauge-fixing conditions introduced in [35].

Since the horizon function displays BRST invariance, it follows immediately that the new Gribov-Zwanziger action

$$
S_{\mathrm{GZ}}^{\prime}=S_{\mathrm{FP}}+S_{h}^{\prime}
$$

is BRST invariant. As a consequence, correlation functions of BRST-invariant operators are independent of gauge parameters, as discussed in [35]. In particular, we can consider the class of Lorentz and color invariant gauges known as the $R_{\xi}$ gauges [7]. Its gauge fixing action is given by

$$
\begin{aligned}
R_{\xi}= & s \int d^{4} x \bar{c}^{a}\left(\partial_{\mu} A_{\mu}^{a}-\mu^{2} \xi^{a}-i \frac{\alpha}{2} b^{a}\right) \\
= & \int d^{4} x\left(i b^{a} \partial_{\mu} A_{\mu}^{a}-\bar{c}^{a} \mathcal{M}^{a b}(A) c^{b}-i \mu^{2} b^{a} \xi^{a}\right. \\
& \left.+\mu^{2} \bar{c}^{a} g^{a b}(\xi) c^{b}+\frac{\alpha}{2} b^{a} b^{a}\right),
\end{aligned}
$$

where $\left(\mu^{2}, \alpha\right)$ are gauge parameters and $\mathcal{M}^{a b}(A)$ is the Faddeev-Popov operator in the $R_{\xi}$ gauge

$$
\mathcal{M}^{a b}(A)=-\partial_{\mu} D_{\mu}^{a b}(A),
$$

where

$$
D_{\mu}^{a b}(A)=\delta^{a b} \partial_{\mu}-g f^{a b c} A_{\mu}^{c}
$$

is the covariant derivative in the adjoint representation of $S U(N)$. The field functional $g^{a b}(\xi)$ is defined in Eq. (A5) in the Appendix A and is derived from the BRST transformation of the Stueckelberg field $\xi$.

Note that the $\mu \rightarrow 0$ limit corresponds to the linear covariant gauge, in which $\alpha \rightarrow 0$ is the Landau gauge. The massive parameter $\mu$ (roughly a ghost mass) works as an infrared regulator for the Stueckelberg field $\xi$. This feature is especially useful for $\alpha \neq 0$. For the Landau gauge, the Stueckelberg field $\xi$ decouples, since all its propagators vanish [32] and no infrared problems involving this auxiliary field appear.

Putting all ingredients in a single action, we obtain the BRST-invariant Gribov-Zwanziger action in the $R_{\xi}$ gauge 


$$
\begin{aligned}
S_{\mathrm{GZ}}= & \int d^{d} x \frac{1}{4} F_{\mu \nu} F_{\mu \nu}+R_{\xi} \\
& +\int d^{d} x\left(\bar{\varphi}_{\mu}^{a c} \mathcal{M}^{a b}\left(A^{h}\right) \varphi_{\mu}^{b c}-\bar{\omega}_{\mu}^{a c} \mathcal{M}^{a b}\left(A^{h}\right) \omega_{\mu}^{b c}\right) \\
& +g \gamma^{2} f^{a b c} \int d^{4} x\left(A^{h}\right)_{\mu}^{a}\left(\varphi_{\mu}^{b c}-\bar{\varphi}_{\mu}^{b c}\right) \\
& +\int d^{4} x\left(i \tau^{a} \partial_{\mu}\left(A^{h}\right)_{\mu}^{a}+\bar{\eta}^{a} \mathcal{M}^{a b}\left(A^{h}\right) \eta^{b}\right) .
\end{aligned}
$$

It is important to point out here that the expression above, Eq. (14), is BRST invariant. As such, the Gribov parameter $\gamma^{2}$ acquires a potential physical meaning, being associated to a nontrivial BRST-invariant quantity, as expressed by

$$
\frac{\partial S_{\mathrm{GZ}}}{\partial \gamma^{2}}=g f^{a b c} \int d^{4} x\left(A^{h}\right)_{\mu}^{a}\left(\varphi_{\mu}^{b c}-\bar{\varphi}_{\mu}^{b c}\right) \neq s \hat{\Delta},
$$

for any local field polynomial $\Delta$. Expression (15) ensures that $\gamma^{2}$ is independent of the gauge parameters $\left(\alpha, \mu^{2}\right)$ entering the gauge $R_{\xi}$ condition.

Finally, let us point out that the auxiliary field $\tau$ in expression (14) is necessary to ensure the transversality of $A^{h}$, a feature that is crucial for the renormalizability of the GZ action (14) [40]. Also, the new couple of ghosts $(\eta, \bar{\eta})$ takes into account the Jacobian arising from the integration over $\tau$ [40].

\section{THE VACUUM ENERGY AT LEADING ORDER IN THE PRESENCE OF CONSTANT SOURCES}

In order to study the dynamical generation of the condensates $\left\langle A^{h} A^{h}\right\rangle$ and $\langle\bar{\varphi} \varphi-\bar{\omega} \omega\rangle$, we introduce the corresponding operators in the GZ action, through constant sources $m^{2}$ and $M^{2}$, so that

$$
\begin{aligned}
S= & S_{G Z}+\frac{m^{2}}{2} \int d^{4} x\left(A^{h}\right)_{\mu}^{a}\left(A^{h}\right)_{\mu}^{a} \\
& +M^{2} \int d^{4} x\left(\bar{\varphi}_{\mu}^{a b} \varphi_{\mu}^{a b}-\bar{\omega}_{\mu}^{a b} \omega_{\mu}^{a b}\right) .
\end{aligned}
$$

In the presence of the sources $m^{2}$ and $M^{2}$, the partition function of the theory reads

$$
Z=\int[D \vec{\phi}] e^{-S}=e^{-V \mathcal{E}_{\text {vac }}}
$$

where $[D \vec{\phi}]=[D A][D b][D \bar{c}][D c][D \bar{\varphi}][D \varphi][D \bar{\omega}][D \omega][D \tau]$ $[D \xi][D \bar{\eta}][D \eta]$ represents the functional measure including all fields present in the action and $\mathcal{E}_{\mathrm{vac}}$ is the energy density of the vacuum state of the GZ theory, in the presence of the source terms, as in Eq. (16). Therefore,

$$
\mathcal{E}_{\text {vac }}=-\frac{1}{V} \log Z=\frac{1}{V} \log \int[D \vec{\phi}] e^{-S} .
$$

In order to show that nonzero condensates appear already at leading order, let us consider the quadratic terms in the action (14),

$$
\begin{aligned}
S_{\text {quadr }}= & \int d^{4} x\left(\frac{1}{4}\left(\partial_{\mu} A_{\nu}^{a}-\partial_{\nu} A_{\mu}^{a}\right)^{2}-\bar{\varphi}_{\mu}^{a b} \partial^{2} \varphi_{\mu}^{a b}+\bar{\omega}_{\mu}^{a b} \partial^{2} \omega_{\mu}^{a b}+g \gamma^{2}\left(A_{\mu}^{a}-\partial_{\mu} \xi^{a}\right) f^{a b c}\left(\varphi_{\mu}^{b c}-\bar{\varphi}_{\mu}^{b c}\right)+i \tau^{a}\left(\partial_{\mu} A_{\mu}^{a}-\partial^{2} \xi^{a}\right)\right. \\
& +\bar{\eta}^{a} \partial^{2} \eta^{a}+i b^{a} \partial_{\mu} A_{\mu}^{a}+\frac{\alpha}{2} b^{a} b^{a}-i \mu^{2} b^{a} \xi^{a}+\bar{c}^{a} \partial^{2} c^{a}-\mu^{2} \bar{c}^{a} c^{a} \\
& \left.+\frac{m^{2}}{2}\left(A_{\mu}^{a}-\partial_{\mu} \xi^{a}\right)\left(A_{\mu}^{a}-\partial_{\mu} \xi^{a}\right)+M^{2}\left(\bar{\varphi}_{\mu}^{a b} \varphi_{\mu}^{a b}-\bar{\omega}_{\mu}^{a b} \omega_{\mu}^{a b}\right)\right) .
\end{aligned}
$$

Within the quadratic approximation (19), the vacuum energy (18) can be calculated by simply integrating out the fields iteratively using the standard formula [2]

$$
\begin{aligned}
& \int[D \phi] \exp \left[-\int d^{d} x \frac{1}{2}(\phi \cdot \hat{A} \cdot \phi)+\int d^{d} x J \cdot \phi\right] \\
& =(\operatorname{det} \hat{A})^{-1 / 2} \exp \left(\frac{1}{2} \int d^{d} x d^{d} y J(x) G_{A}(x, y) J(y)\right),
\end{aligned}
$$

It is convenient to start with the auxiliary fields $\tau, \bar{\eta}$ and $\eta$, which are related to the constraint on the transversality of $A^{h}$. Next, one may integrate out the Stueckelberg field $\xi$ and then Zwanziger's auxiliary fields $\bar{\varphi}, \varphi, \bar{\omega}$ and $\omega$, followed by the gauge fixing fields $b, \bar{c}$ and $c$. At the end of this procedure, we are left with an integral over the gauge field $A$

$$
Z=\int[D A] \operatorname{det}(\alpha)^{-1 / 2} \operatorname{det}\left(-\partial^{2}+\mu^{2}\right) e^{-S_{A}}
$$

where $G_{A}$ is the Green's function of the operator $\hat{A}$. where 


$$
\begin{aligned}
S_{A}= & \frac{1}{2} \int d^{d} x A_{\mu}^{a} \delta^{a b}\left[\left(-\partial^{2}+m^{2}+\frac{2 g^{2} N_{c} \gamma^{4}}{-\partial^{2}+M^{2}}\right) P_{\mu \nu}\right. \\
& \left.-\frac{1}{\alpha}\left(1+\frac{\mu^{2}}{-\partial^{2}}\right)^{2} \partial_{\mu} \partial_{\nu}\right] A_{\nu}^{b}
\end{aligned}
$$

with the transverse projector

$$
P_{\mu \nu}=\delta_{\mu \nu}-\frac{\partial_{\mu} \partial_{\nu}}{\partial^{2}}
$$

After the final integration in the gluon field, the gaugedependent terms present in the longitudinal part of the gluon action (22) precisely cancel the determinants in (21) and the vacuum energy density is, in the quadratic approximation,

$$
\begin{aligned}
\mathcal{E}_{\text {vac }}^{\text {(quad) }}= & \frac{N_{c}^{2}-1}{2}(d-1) \int \frac{d^{d} p}{(2 \pi)^{d}} \log \left(p^{2}+m^{2}+\frac{\Lambda^{4}}{p^{2}+M^{2}}\right) \\
& -\frac{N_{c}^{2}-1}{2} \int \frac{d^{d} p}{(2 \pi)^{d}} \log p^{2}
\end{aligned}
$$

where $\Lambda^{4}=2 g^{2} N_{c} \gamma^{4}$. Let us remark that the independence of (24) with respect to the gauge parameters $\alpha$ and $\mu^{2}$ is an explicit consequence of the exact BRST invariance of the present formulation of the Gribov-Zwanziger framework (14). Note that this property would not be true for the original Gribov-Zwanziger framework, in which the BRST symmetry is softly broken. This is the first important feature of the proposed action (14) that we wish to show in this paper.

\section{TWO GAUGE INVARIANT CONDENSATES AND THE INSTABILITY OF THE GZ ACTION}

Now that we have calculated the vacuum energy density in the quadratic approximation, we may proceed to calculate the condensates

$$
\left\langle\left(A^{h}\right)_{\mu}^{a}\left(A^{h}\right)_{\mu}^{a}\right\rangle=\frac{1}{Z} \int[D \vec{\Phi}]\left(A^{h}\right)_{\mu}^{a}\left(A^{h}\right)_{\mu}^{a} e^{-S_{\mathrm{GZ}}}
$$

and

$\left\langle\bar{\varphi}_{\mu}^{a b} \varphi_{\mu}^{a b}-\bar{\omega}_{\mu}^{a b} \omega_{\mu}^{a b}\right\rangle=\frac{1}{Z} \int[D \vec{\Phi}]\left(\bar{\varphi}_{\mu}^{a b} \varphi_{\mu}^{a b}-\bar{\omega}_{\mu}^{a b} \omega_{\mu}^{a b}\right) e^{-S_{\mathrm{GZ}}}$

Note that these condensates are calculated within the BRST invariant formulation of the Gribov-Zwanziger theory, hence the action $S_{G Z}$, Eq. (14), in the definitions above. An important property of both condensates is that they correspond to expectation values of BRST invariant operators, as briefly reviewed in Appendix A. They can furthermore be expressed in terms of the vacuum energy as

$$
\left\langle\left(A^{h}\right)_{\mu}^{a}\left(A^{h}\right)_{\mu}^{a}\right\rangle=\left.2 \frac{\partial \mathcal{E}_{\mathrm{vac}}}{\partial m^{2}}\right|_{m^{2}=M^{2}=0}
$$

and

$$
\left\langle\bar{\varphi}_{\mu}^{a b} \varphi_{\mu}^{a b}-\bar{\omega}_{\mu}^{a b} \omega_{\mu}^{a b}\right\rangle=\left.\frac{\partial \mathcal{E}_{\mathrm{vac}}}{\partial M^{2}}\right|_{m^{2}=M^{2}=0} .
$$

Note that the sources $m^{2}$ and $M^{2}$ are taken to zero at the end of the calculation. This is analogous to considering the appearance of a spontaneous magnetization in a spin model: first, one considers the interaction of the magnetic moments in the presence of some external magnetic field and then, at the last step of the calculation, the external field is taken to zero. A resulting nonzero value of the condensates $\left\langle A^{h} A^{h}\right\rangle$ or $\langle\bar{\varphi} \varphi-\bar{\omega} \omega\rangle$ starting from the GZ action (14) is somewhat analogous to a spontaneous magnetization in a spin model [41].

Even though our calculation already shows that the condensates $\left\langle A^{h} A^{h}\right\rangle$ and $\langle\bar{\varphi} \varphi-\bar{\omega} \omega\rangle$ are nonzero, a fuller calculation would require the construction of effective potential for the expectation values of these two operators, a task which can be faced by means of the local composite operator (LCO) formalism [42,43], see [30,44-46] for previous attempts in the context of the RGZ theory in the Landau gauge and [47] for recent developments.

From the expression (24) for the vacuum energy and formulas (27) and (28), the condensates can be immediately calculated, leading to

$$
\begin{aligned}
\left\langle\left(A^{h}\right)_{\mu}^{a}\left(A^{h}\right)_{\mu}^{a}\right\rangle & =\frac{\left(N_{c}^{2}-1\right)(d-1)}{2} \int \frac{d^{d} p}{(2 \pi)^{d}} \frac{1}{p^{2}+\frac{\Lambda^{4}}{p^{2}}} \\
& =-\frac{\left(N_{c}^{2}-1\right)(d-1)}{2} I_{d}(\Lambda)
\end{aligned}
$$

and

$$
\begin{aligned}
& \left\langle\bar{\varphi}_{\mu}^{a b} \varphi_{\mu}^{a b}-\bar{\omega}_{\mu}^{a b} \omega_{\mu}^{a b}\right\rangle \\
& \quad=\left.\frac{\left(N_{c}^{2}-1\right)(d-1)}{2} \int \frac{d^{d} p}{(2 \pi)^{d}} \frac{p^{2}}{p^{4}+\Lambda^{4}} \frac{-\Lambda^{4}}{\left(p^{2}+M^{2}\right)^{2}}\right|_{M=0} \\
& \quad=-\frac{\left(N_{c}^{2}-1\right)(d-1)}{2} I_{d}(\Lambda)
\end{aligned}
$$

where we used the decomposition

$$
\frac{1}{p^{2}+\frac{\Lambda^{4}}{p^{2}}}=\frac{1}{p^{2}}-\frac{\Lambda^{4}}{p^{2}\left(p^{4}+\Lambda^{4}\right)}
$$

and the dimensional regularization formula

$$
\int \frac{d^{d} p}{(2 \pi)^{d}} \frac{1}{p^{2}}=0
$$


which is valid for $d>2$. For the final expressions, we also used the results

$$
\begin{array}{rlrl}
I_{d}(\Lambda):=\int \frac{d^{d} p}{(2 \pi)^{d}} \frac{\Lambda^{4}}{p^{2}\left(p^{4}+\Lambda^{4}\right)} & =\frac{\Lambda^{2}}{32 \pi} & & (d=4) \\
& =\frac{\sqrt{2} \Lambda}{8 \pi} & & (d=3) \\
& \rightarrow \infty & (d=2) .
\end{array}
$$

Note that, for $d=2$, the integral (33) is IR divergent, so that the condensates do not show up, as could be anticipated by the Mermin-Wagner-Coleman theorem [48,49]. In $d=4$ and in $d=3$, these leading-order results for the condensates vanish if one does not consider the restriction to the Gribov region, i.e., if $\gamma=0$ (or, equivalently, $\Lambda=0$ ). It is important to note that it has been shown in pure YangMills theories in Landau gauge that the $\left\langle A^{2}\right\rangle$ condensate is generated by the non-Abelian interaction, even in the absence of the Gribov horizon [44,45]. This finding is compatible with our quadratic analysis because in our approximation we neglect the direct effect of interactions, considering only the presence of the nonperturbative background brought about by the constraint of the Gribov horizon condition. A full fledged effective potential calculation would therefore reveal that the $\left\langle\left(A^{h}\right)_{\mu}^{a}\left(A^{h}\right)_{\mu}^{a}\right\rangle$ condensate has a nonzero limit as the Gribov parameter vanishes.

Let us finally note that the Gribov parameter is independent of the gauge parameters and is thus allowed to enter explicitly findings for physical observables. This can be immediately seen from the defining equation of $\gamma$, the gap equation [12]

$$
\frac{\partial \mathcal{E}_{\mathrm{vac}}}{\partial \gamma^{2}}=0
$$

where $\mathcal{E}_{\text {vac }}$ is the vacuum energy (24), which is explicitly independent of the gauge parameters $\alpha$ and $\mu$.

Due to the BRST invariance, it turns out that the condensates $\left\langle\left(A^{h}\right)_{\mu}^{a}\left(A^{h}\right)_{\mu}^{a}\right\rangle$ and $\left\langle\bar{\varphi}_{\mu}^{a b} \varphi_{\mu}^{a b}-\bar{\omega}_{\mu}^{a b} \omega_{\mu}^{a b}\right\rangle$, (29) and (30), are also independent of the gauge parameters $\alpha$ and $\mu$ appearing in the gauge condition. This is a direct consequence of the BRST-invariant formulation of the Gribov-Zwanziger theory put forward in $[31,32,38]$. It is also interesting to note that, due to the independence of the energy density on the gauge parameters, if the condensation of these operators happens for some value of $\alpha$ and $\mu$ (for example, for the Landau gauge), it will happen for any values of the gauge parameters. This property indicate that indeed these condensates may have a genuine physical meaning. Furthermore, the RGZ parameters $m^{2}$ and $M^{2}$ related to the condensates do not depend on the gauge parameters and are thus given by their Landau gauge values. Finally, although the gluon propagator $\left\langle A_{\mu}^{a}(p) A_{\nu}^{b}(-p)\right\rangle$ is gauge dependent, the two-point correlation point of the $A^{h}$ composite field is gauge independent, being equal to the Landau gauge gluon propagator. Thus, it is possible to use this idea to predict how the gluon propagator deviates from the Landau gauge value as one considers nonzero values for the gauge parameters $\alpha$ and $\mu$ for some generic $R_{\xi}$ gauges.

\section{FINAL REMARKS}

In this work, we have extended previous discussions on the generation of dimension-two condensates in the Landau gauge Gribov-Zwanziger framework to a two-parameter family of gauges, namely, the $R_{\xi}$ gauges, parametrized by the parameters $\alpha$ and $\mu$. In the limit $\mu \rightarrow 0$, one recovers the linear covariant gauge, so that with $(\mu, \alpha) \rightarrow 0$ one achieves the Landau gauge. We have explicitly shown that the instability of the GZ action observed in the Landau gauge is actually a universal property in this large class of gauges, suggesting a genuine physical meaning for the refinement of the GZ action in the infrared regime. In particular, we have shown that the GZ action is unstable with respect to the formation of the BRST invariant condensates $\left\langle\left(A^{h}\right)_{\mu}^{a}\left(A^{h}\right)_{\mu}^{a}\right\rangle$ and $\left\langle\bar{\varphi}_{\mu}^{a b} \varphi_{\mu}^{a b}-\bar{\omega}_{\mu}^{a b} \omega_{\mu}^{a b}\right\rangle$, which were computed at leading order. These condensates are proportional to the Gribov parameter $\gamma^{2}$, which is also independent of the choice of the gauge parameters contained in the $R_{\xi}$ gauges.

Such gauge independence reinforces the fact that both Gribov parameter and condensates enter the correlation functions of physical operators, i.e., the correlation functions of local gauge invariant quantity as, for example, the glueball spectrum (cf. e.g., [50,51]). The results presented here are therefore a step forward in proving that the RGZ framework can provide a physically meaningful description of the infrared regime of Yang-Mills theories.

An interesting point raised by these developments is whether one can probe directly these gauge-invariant dimension-two condensates on the lattice. For that, one would need to write these nonlocal operators in terms of lattice variables, a task which is not straightforward. Nevertheless, as we have emphasized, the current description of the RGZ formalism in $R_{\xi}$ gauges implies that the numerical results for expectation values of BRST-invariant operators must be identical to those in the Landau gauge, which is already accessible by lattice methods. Indeed there are indications of a nonzero $\left\langle A^{2}\right\rangle$ in lattice Yang-Mills simulations for the Landau gauge $[52,53]$ and we expect to report on direct comparisons between the RGZ predictions and the lattice results in the future.

\section{ACKNOWLEDGMENTS}

The authors would like to thank the Brazilian agencies Conselho Nacional de Desenvolvimento Científico e Tecnológico $(\mathrm{CNPq})$ and Fundação Carlos Chagas de Amparo à Pesquisa do Estado do Rio de Janeiro (FAPERJ) for financial support. This paper is also part of 
the project Instituto Nacional de Ciência e TecnologiaFísica Nuclear e Aplicações (INCT-FNA) Process No. 464898/2014-5. B. W. M. is supported by CNPq project Universal (Grant No. 431796/2016-5) and FAPERJ (Grant No. E-26/202.649/2018). This study was financed in part by the Coordenação de Aperfeiçoamento de Pessoal de Nível Superior-Brasil (CAPES)-Financial Code 001 (M. N. F.).

\section{APPENDIX: THE BRST TRANSFORMATIONS AND THE GAUGE INVARIANCE OF THE GZ ACTION}

For completeness, let us introduce the BRST transformations of the fields present in the GZ action (14) in order to show that such an action [as well as its version with sources, Eq. (16)] is BRST invariant. The BRST transformations of the fields are

$$
\begin{aligned}
s A_{\mu}^{a} & =-D_{\mu}^{a b} c^{b} \\
s c^{a} & =\frac{g}{2} f^{a b c} c^{b} c^{c} \\
s \bar{c}^{a} & =i b^{a} \\
s b^{a} & =0,
\end{aligned}
$$

as in standard perturbative Yang-Mills theories, supplemented by the trivial transformations of the auxiliary fields

$$
s \Phi=0,
$$

where $\Phi$ is any of the fields $\bar{\varphi}, \varphi, \bar{\omega}, \omega, \tau, \bar{\eta}, \eta$. The field $A^{h}$ is, by construction, BRST invariant, so that

$$
s\left(A^{h}\right)_{\mu}^{a}=0 .
$$

From the definition (7) of $A^{h}$, the condition (A3) leads to the particular BRST transformation of the Stueckelberg field $\xi$ [32]

$$
s \xi^{a}=g^{a b} c^{b}
$$

where

$$
g^{a b}(\xi)=-\delta^{a b}+\frac{g}{2} f^{a b c} \xi^{c}-\frac{g^{2}}{12} f^{a m r} f^{m b q} \xi^{q} \xi^{r}+\mathcal{O}\left(\xi^{3}\right) .
$$

As is well-known, the Faddeev-Popov action

$$
S_{\mathrm{FP}}=\int d^{d} x \frac{1}{4} F_{\mu \nu} F_{\mu \nu}+R_{\xi},
$$

is invariant under the BRST transformations (A1), where $R_{\xi}$ is the gauge-fixing action in (11). Furthermore, with the BRST transformations (A2) and (A3) one can immediately check that the Gribov-Zwanziger action (14) is invariant under BRST transformations, as advertised.

Finally, note that the addition of the BRST-invariant sources $\left\langle\left(A^{h}\right)_{\mu}^{a}\left(A^{h}\right)_{\mu}^{a}\right\rangle$ and $\left\langle\bar{\varphi}_{\mu}^{a b} \varphi_{\mu}^{a b}-\bar{\omega}_{\mu}^{a b} \omega_{\mu}^{a b}\right\rangle$ does not spoil the BRST invariance of the action (16).
[1] L. D. Faddeev and V. N. Popov, Phys. Lett. 25B, 29 (1967).

[2] M. E. Peskin and D. V. Schroeder, An Introduction to Quantum Field Theory (Addison-Wesley, Reading, MA, 1995).

[3] S. Weinberg, The Quantum Theory of Fields Vol. 1: Foundations (Cambridge University Press, Cambridge, England, 2005).

[4] D. Zwanziger, Phys. Rev. Lett. 94, 182301 (2005).

[5] M. S. Guimaraes, B. W. Mintz, and S. P. Sorella, Phys. Rev. D 91, 121701 (2015).

[6] D. R. Campagnari, E. Ebadati, H. Reinhardt, and P. Vastag, Phys. Rev. D 94, 074027 (2016).

[7] G. 't Hooft, Nucl. Phys. B190, 455 (1981).

[8] A. S. Kronfeld, M. L. Laursen, G. Schierholz, and U. J. Wiese, Phys. Lett. B 198, 516 (1987).

[9] A. S. Kronfeld, G. Schierholz, and U. J. Wiese, Nucl. Phys. B293, 461 (1987).

[10] P. Gaigg, W. Kummer, and M. Schweda, Physical and Nonstandard Gauges, Lecture Notes in Physics (Springer, Switzerland, 1990), Vol. 361.

[11] K. G. Wilson, Phys. Rev. D 10, 2445 (1974).

[12] V. N. Gribov, Nucl. Phys. B139, 1 (1978).
[13] An analogous argument can also be cast in the other mentioned gauges.

[14] D. Zwanziger, Nucl. Phys. B323, 513 (1989).

[15] N. Vandersickel and D. Zwanziger, Phys. Rep. 520, 175 (2012).

[16] K. Osterwalder and R. Schrader, Commun. Math. Phys. 31, 83 (1973).

[17] K. Osterwalder and R. Schrader, Commun. Math. Phys. 42, 281 (1975).

[18] R. Oehme, Int. J. Mod. Phys. A 10, 1995 (1995).

[19] K. Nishijima, Czech. J. Phys. 46, 1 (1996).

[20] R. Alkofer and L. von Smekal, Phys. Rep. 353, 281 (2001).

[21] A. Cucchieri and T. Mendes, Phys. Rev. Lett. 100, 241601 (2008).

[22] C. S. Fischer, A. Maas, and J. M. Pawlowski, Ann. Phys. (Amsterdam) 324, 2408 (2009).

[23] O. Oliveira and P. J. Silva, Phys. Rev. D 79, 031501 (2009).

[24] A. Cucchieri and T. Mendes, Phys. Rev. D 78, 094503 (2008).

[25] D. Dudal, S. P. Sorella, N. Vandersickel, and H. Verschelde, Phys. Rev. D 77, 071501 (2008). 
[26] D. Dudal, J. A. Gracey, S. P. Sorella, N. Vandersickel, and H. Verschelde, Phys. Rev. D 78, 065047 (2008).

[27] A. C. Aguilar, D. Binosi, and J. Papavassiliou, Phys. Rev. D 78, 025010 (2008).

[28] P. Boucaud, J.-P. Leroy, A. L. Yaouanc, J. Micheli, O. Pene, and J. Rodriguez-Quintero, J. High Energy Phys. 06 (2008) 012.

[29] A. K. Cyrol, L. Fister, M. Mitter, J. M. Pawlowski, and N. Strodthoff, Phys. Rev. D 94, 054005 (2016).

[30] D. Dudal, S. P. Sorella, and N. Vandersickel, Phys. Rev. D 84, 065039 (2011).

[31] M. A. L. Capri, D. Dudal, D. Fiorentini, M. S. Guimaraes, I. F. Justo, A. D. Pereira, B. W. Mintz, L. F. Palhares, R. F. Sobreiro, and S. P. Sorella, Phys. Rev. D 92, 045039 (2015).

[32] M. A. L. Capri, D. Dudal, D. Fiorentini, M. S. Guimaraes, I. F. Justo, A. D. Pereira, B. W. Mintz, L. F. Palhares, R. F. Sobreiro, and S. P. Sorella, Phys. Rev. D 94, 025035 (2016).

[33] M. A. L. Capri, D. Dudal, A. D. Pereira, D. Fiorentini, M. S. Guimaraes, B. W. Mintz, L. F. Palhares, and S. P. Sorella, Phys. Rev. D 95, 045011 (2017).

[34] M. A. L. Capri, D. Fiorentini, A. D. Pereira, and S. P. Sorella, Eur. Phys. J. C 77, 546 (2017).

[35] M. A. L. Capri, D. Dudal, M. S. Guimaraes, A. D. Pereira, B. W. Mintz, L. F. Palhares, and S. P. Sorella, Phys. Lett. B 781, 48 (2018).

[36] R. Delbourgo and G. Thompson, Phys. Rev. Lett. 57, 2610 (1986).

[37] M. Lavelle and D. McMullan, Phys. Rep. 279, 1 (1997).

[38] M. A. L. Capri, D. Fiorentini, M. S. Guimaraes, B. W. Mintz, L. F. Palhares, S. P. Sorella, D. Dudal, I. F. Justo, A. D. Pereira, and R. F. Sobreiro, Phys. Rev. D 93, 065019 (2016).
[39] M. A. L. Capri, D. Fiorentini, M. S. Guimaraes, B. W. Mintz, L. F. Palhares, and S. P. Sorella, Phys. Rev. D 94, 065009 (2016).

[40] M. A. L. Capri, D. Fiorentini, A. D. Pereira, and S. P. Sorella, Phys. Rev. D 96, 054022 (2017).

[41] With an important difference that the operators which condense in our case are not elementary fields that enter the Lagrangian, as would be the case for spontaneous magnetization in a spin model.

[42] H. Verschelde, K. Knecht, K. Van Acoleyen, and M. Vanderkelen, Phys. Lett. B 516, 307 (2001).

[43] K. Knecht and H. Verschelde, Phys. Rev. D 64, 085006 (2001).

[44] D. Dudal, H. Verschelde, R. E. Browne, and J. A. Gracey, Phys. Lett. B 562, 87 (2003).

[45] R. E. Browne and J. A. Gracey, J. High Energy Phys. 11 (2003) 029.

[46] D. Dudal, R. F. Sobreiro, S. P. Sorella, and H. Verschelde, Phys. Rev. D 72, 014016 (2005).

[47] D. Dudal, C. Felix, L. Palhares, F. Rondeau, and D. Vercauteren, arXiv:1811.12524.

[48] N. D. Mermin and H. Wagner, Phys. Rev. Lett. 17, 1133 (1966).

[49] S. R. Coleman, Commun. Math. Phys. 31, 259 (1973).

[50] D. Dudal, M. S. Guimaraes, and S. P. Sorella, Phys. Lett. B 732, 247 (2014).

[51] D. Dudal, M. S. Guimaraes, and S. P. Sorella, Phys. Rev. Lett. 106, 062003 (2011).

[52] O. Pene et al., Proc. Sci., FACESQCD2010 (2010) 010.

[53] M. N. Chernodub and E. M. Ilgenfritz, Phys. Rev. D 78, 034036 (2008). 